\title{
Prevalencia y Severidad de Fluorosis Dental y su Aso- ciación con Historia de Caries en Escolares que Con- sumen Agua Potable Fluorurada en Temuco, Chile
}

\author{
Prevalence and Severity of Dental Fluorosis and its Association with History of Dental \\ Caries in Schoolchildren Consuming Fluoridated Drinking Water in Temuco, Chile
}

Denise Olivares-Keller; ; María José Arellano-Valeria*; Jorge Cortés ${ }^{* *}$ \& Mario Cantín ${ }^{* * * * * * * *}$

OLIVARES-KELLER, D.; ARELLANO-VALERIA, M. J.; CORTÉS, J. \& CANTÍN, M. Prevalencia y severidad de fluorosis dental y su asociación con historia de caries en escolares que consumen agua potable fluorurada en Temuco, Chile. Int. J. Odontostomat., 7(3):447-454, 2013.

RESUMEN: La fluoruración del agua potable comenzó a implementarse en Chile en la década de 1950 para prevenir el principal problema de salud oral en la población, la caries dental. Se ha aplicado sistemáticamente en el país, y en Temuco desde el año 2004. Sin embargo, la ingesta de fluoruros en periodos críticos del desarrollo dentario de manera excesiva produce fluorosis dental. El objetivo de este estudio fue determinar la prevalencia y severidad de la fluorosis dental en escolares de segundo básico que consumen agua potable fluorurada en la ciudad de Temuco, Chile y asociarla con la historia de caries. Se realizó un estudio epidemiológico descriptivo y explicativo con un muestreo aleatorio estratificado de la población escolar de 7 años que cursaba segundo básico durante el año 2012 en colegios particulares, subvencionados y municipales de Temuco. Sobre una muestra de 317 niños, se determinó el índice Dean y la historia de caries. La prevalencia de fluorosis dental fue 53,31\% (169 niños), y la severidad fue de tipo cuestionable, muy leve y leve en el 31,36\%, 42,6\% y $22,4 \%$ de los casos, respectivamente. El 3,5\% fue moderado. No se observó asociación significativa entre fluorosis e historia de caries. En Temuco, la prevalencia de fluorosis dental en niños de 7 años es alta, con una severidad de tipo cuestionable, muy leve y leve en más del $50 \%$ de los casos. Luego de 8 años de implementar la fluoruración del agua potable, hubo una disminución del daño acumulado por caries, pero la fluorosis dental aumentó en la población.

PALABRAS CLAVE: fluorosis dental, grado de fluorosis, caries dental, agua potable, índice de Dean.

\section{INTRODUCCIÓN}

La caries dental es el principal problema de salud pública oral en Chile, con una prevalencia según el Diagnóstico de Salud Bucal realizado por el Ministerio de Salud del $70,36 \%$ en escolares de 6 años (Soto et al., 2007).

Dentro de las estrategias utilizadas para prevenir el desarrollo y progresión de la caries, el uso de flúor (fluoruro) ha demostrado científicamente su eficacia (de Carvalho et al., 2011). En este contexto, surgió la fluoruración de alimentos de alto consumo poblacional como la sal, la leche y el agua potable.
La fluoruración del agua potable es una acción de carácter masivo, equitativo y de alto impacto; ha sido implementada por diversos países incluido Chile desde la década de 1950 (estudio piloto), cuando la Asociación Dental Americana (ADA) recomendó esta medida para la prevención de caries en la población de Estados Unidos, apoyada posteriormente por la Organización Mundial de la Salud (OMS) y Organización Panamericana de la Salud (OPS).

Sin embargo la ingesta excesiva de fluoruros puede ocasionar fluorosis dental, un defecto irreversi-

\footnotetext{
Escuela de Odontología, Facultad de Odontología, Universidad de La Frontera, Temuco, Chile.

** Departamento de Matemática y Estadística, Universidad de la Frontera, Temuco, Chile.

*** Programa de Doctorado en Ciencias Morfológicas. CIMA, Facultad de Odontología, Universidad de La Frontera, Temuco, Chile.

${ }^{* * * * *}$ Centro de Investigación en Ciencias Biomédicas, Universidad Autónoma de Chile, Temuco, Chile.
} 
ble en la formación del esmalte que resulta del consumo excesivo y prolongado de fluoruros. La exposición a fluoruros entre los 15-30 meses de edad, se considera un periodo crítico para el desarrollo de fluorosis en los dientes permanentes con mayor importancia estética, aunque en edades más tempranas también es preocupante (Levy, 2003). Durante el periodo de amelogénesis, los dientes son vulnerables a los efectos del fluoruro, afectando la conformación de los cristales de hidroxiapatita hacia fluor-hidroxiapatita, donde el riesgo de fluorosis está más relacionado con la exposición total acumulada al fluoruro durante el desarrollo de la dentición que a una exposición limitada a periodos específicos.

En los dientes definitivos afectados por fluorosis dental, los signos pueden ir desde manchas de color blanquecino hasta un café oscuro, e incluso llegar a la pérdida de continuidad del esmalte de acuerdo a la severidad. En dientes primarios es infrecuente y más leve (Aoba \& Fejerskov, 2002).

La prevalencia de fluorosis dental parece seguir una tendencia opuesta a la prevalencia de caries, con índices crecientes reportados en todo el mundo, su severidad se ha manifestado principalmente en sus formas leve y muy leve según el índice de Dean, lo que se observa tanto en comunidades con agua fluorada como no fluorada (Buzalaf \& Levy, 2011).

En Chile la fluoruración del agua potable comenzó a aplicarse de manera sistemática en la ciudad de Valparaíso en la década de 1980, y hasta el año 2011 ha alcanzado una cobertura poblacional del $72 \%$. En la ciudad de Temuco, la medida fue implementada el año 2004 y hasta la fecha no existen estudios epidemiológicos publicados en revistas científicas, en el contexto de fluorosis dental para esta ciudad.

El objetivo de este estudio fue evaluar la prevalencia y severidad de la fluorosis dental en escolares de segundo básico que consumen agua potable fluorurada en la ciudad de Temuco, Chile, y asociarla con la historia de caries.

\section{MATERIAL Y MÉTODO}

Diseño. Se realizó un estudio epidemiológico descriptivo y explicativo, de corte transversal en una muestra aleatoria de la población de escolares de 7 años que cursaban segundo año básico (educación primaria), pertenecientes a establecimientos educacionales de tipo particular, subvencionado y municipal, todos de carácter urbano en la comuna de Temuco. El estudio fue aprobado por el comité de Ética de la Facultad de Medicina, Universidad de La Frontera.

Población y Selección de la Muestra. El Universo de la población en estudio estuvo constituida por 3.635 alumnos que cursaron segundo básico durante el año 2012; correspondiente a colegios municipales (956 alumnos), establecimientos subvencionados con financiamiento compartido (2.335 alumnos) y colegios particulares pagados (344 alumnos), todos surtidos de agua potable fluorurada. La información poblacional se encuentra registrada en el Departamento de Bioestadística de la Dirección Provincial de Educacional, IX Región de la Araucanía, Temuco, Chile. El tamaño de la muestra fue estimado a través de un muestreo aleatorio simple estratificado mediante el software SPSS versión 20, con error máximo del $5 \%$ y confianza del $95 \%$ para alcanzar una muestra de 341 niños.

A los niños, profesores y directores de los establecimientos educacionales se les informó detalladamente en qué consistía el estudio, y los padres y/o representantes de los niños fueron contactados e informados en una carta sobre el diseño del examen dental que se realizaría a sus hijos, a la vez que, en caso de aceptar formar parte en el estudio, se requería la firma de una carta de consentimiento informado. Fueron incluidos los niños que asintieran participar y contaran con el consentimiento de los padres y/o representantes legales, además de tener presentes y totalmente erupcionados en boca los cuatro primeros molares permanentes. Se excluyeron colegios con educación especial, niños con trastornos del comportamiento y/o mentales, a fin de evitar condiciones que pudieran afectar la medición, y niños que estuvieran bajo tratamiento de ortodoncia.

La muestra final se constituyó por 317 niños, 182 mujeres y 135 hombres, con una afijación proporcional para cada uno de los subgrupos, diferenciando 75 sujetos de establecimientos municipales (32 mujeres y 43 hombres), 211 de establecimientos particulares subvencionados (93 mujeres y 118 hombres) y 31 de establecimientos particulares pagados (10 mujeres y $21 \mathrm{hom}$ bres). El detalle de los colegios y número de alumnos seleccionados se observa en la Tabla I. En cada establecimiento se trabajó con cursos completos comenzando por la letra A del curso y sucesivamente hasta cumplir con la cantidad necesaria de alumnos. 
OLIVARES-KELLER, D.; ARELLANO-VALERIA, M. J.; CORTÉS, J. \& CANTíN, M. Prevalencia y severidad de fluorosis dental y su asociación con historia de caries en escolares que consumen agua potable fluorurada en Temuco, Chile. Int. J. Odontostomat., 7(3):447-454, 2013.

Tabla I. Colegios y número de alumnos de 7 años que cursan segundo año básico en colegios de Temuco seleccionados para el estudio.

\begin{tabular}{|c|c|c|c|}
\hline Tipo & Nombre & $\mathbf{n}$ & Total \\
\hline \multirow{3}{*}{ Municipal } & Campos Deportivos & 39 & \multirow{3}{*}{75} \\
\hline & Escuela Standard & 15 & \\
\hline & $\begin{array}{l}\text { Complejo Educacional } \\
\text { Un Amancer En La Araucanía }\end{array}$ & 21 & \\
\hline \multirow{5}{*}{ Subvencionados } & Liceo Augusto Winter & 37 & \multirow{5}{*}{211} \\
\hline & Escuela Madre Admirable & 27 & \\
\hline & Escuela Francia & 29 & \\
\hline & Instituto Claret & 70 & \\
\hline & Colegio Claudio Matte & 48 & \\
\hline \multirow{2}{*}{ Particular } & George Chaytor English College & 14 & \multirow{2}{*}{31} \\
\hline & Colegio Pumahue & 17 & \\
\hline
\end{tabular}

Recolección de datos. En cada establecimiento educacional seleccionado, se acondicionó una sala de clases para realizar los exámenes dentales siguiendo los criterios estandarizados por la OMS. Dos examinadores capacitados y previamente calibrados con un grupo piloto (kappa 0,9) realizaron el examen. El número de sesiones utilizadas en cada establecimiento dependió de la disposición de tiempo asignado y el número de alumnos. Se confeccionó una ficha de recolección de datos para el estudio. Las variables incluidas en el estudio fueron el sexo, tipo de establecimiento educacional, Índice de Higiene Oral Simplificado (IHOS), historia de caries a través de los índices COPD y ceod y grado de severidad de fluorosis a través del índice de Dean (tradicional) (Azpeitia-Valadez et al., 2008; Yévenes et al., 2011; Narwaria \& Saksena, 2013).

Al finalizar la recolección de datos, en los segundos básicos de cada establecimiento educacional se realizaron sesiones educativas sobre salud e higiene oral, y a los padres de los niños examinados se les envió un informe de las condiciones clínicas y dentales encontradas en sus hijos para su conocimiento y que ellos pudieran evaluar la posibilidad de tratarlas según sea el caso.

Análisis estadístico. Los datos recopilados se analizaron mediante estadística descriptiva y análisis de varianza mediante la prueba ANOVA para establecer las Medias y EE. Además, se aplicó un análisis de regresión multivariado para evaluar si existe correlación entre el índice coed en los niños y el índice de Dean, de higiene oral, tipo de colegio estudiado y el sexo de los participantes con el modelo índice coed $=(\mathrm{b} 0+$ b1lHOS + b2Índice de Dean + d1Sexo1 + I1Colegio1
+ 12Colegio2). Además, en función de explicar la severidad de la fluorosis, se realizó un análisis de covarianza para explicar la variable Índice de Dean y las covariables sexo, tipo de colegio (municipalizado, subvencionado y particular) e IHOS con el modelo: Índice de Dean $=\mathrm{f}$ (sexo, tipo de colegio, $\mathrm{IHOS}$ )+Error. Se utilizó un intervalo de confianza del $95 \%$ y un valor de significación estadística $p<0,05$.

\section{RESULTADOS}

La prevalencia de fluorosis dental en los niños estudiados fue de $53,31 \%$ (Tabla II). El grado de severidad observado fue cuestionable en un $31,36 \%$ de los casos, muy leve en $42,6 \%$, leve en $22,4 \%$ y sólo el $3,5 \%$ fue moderado. No se encontraron niños afectados con un nivel severo de fluorosis. El análisis de covarianza demostró que ninguno de los factores, interacción y variable fueron significativos para explicar algún tipo de asociación con el Índice de Dean.

Al analizar el índice ceod, la Media para los 317 casos fue 2,36. En la Tabla III se observan los resultados descriptivos según sexo y tipo de colegio analizado. En el análisis de varianza (se asumen efectos fijos) del índice ceod, la interacción entre sexo y tipo de colegio no fue significativa. Además, la covariable IHOS no fue significativa para explicar las diferencias del índice ceod, solo se encontraron diferencias significativas según el tipo de colegio $(p=0,0086)$ (Tabla IV y Fig. 1). En el índice COPD, la Media para el total de la muestra fue 0,158. En el análisis de varianza el índice COPD no muestra interacción entre ninguna de las variables estudiadas (Tabla $\mathrm{V}$ ). 
OLIVARES-KELLER, D.; ARELLANO-VALERIA, M. J.; CORTÉS, J. \& CANTíN, M. Prevalencia y severidad de fluorosis dental y su asociación con historia de caries en escolares que consumen agua potable fluorurada en Temuco, Chile. Int. J. Odontostomat., 7(3):447-454, 2013.

Tabla II. Prevalencia y grado de severidad de fluorosis dental en la población escolar de niños de ambos sexos que cursan segundo básico en Temuco.

\begin{tabular}{|c|c|c|c|c|}
\hline Índice & Frecuencia & Frec. Rel. & Frec. Acum. & Frec. Rel. Acum. \\
\hline Normal & 148 & 0,466 & 148 & 0,466 \\
\hline Cuestionable & 53 & 0,167 & 201 & 0,634 \\
\hline Muy leve & 72 & 0,227 & 273 & 0,861 \\
\hline Leve & 38 & 0,119 & 311 & 0,981 \\
\hline Moderado & 6 & 0,018 & 317 & 1,000 \\
\hline Severo & 0 & 0 & 317 & 1,000 \\
\hline
\end{tabular}

Tabla III. Resumen de Medias por Mínimos Cuadrados y EE (Error estándar) e intervalos de confianza al 95\%, para el índice ceod.

\begin{tabular}{lccccc}
\hline Variable & Casos & Media & EE & Límite Inf. & Límite Sup. \\
\hline $\begin{array}{lcccc}\text { Sexo } \\
\text { Femenino (F) }\end{array}$ & 135 & 2,230 & 0,316 & 1,608 & 2,852 \\
Masculino (M) & 182 & 2,505 & 0,235 & 2,041 & 2,969 \\
Tipo de Colegio & & & & & \\
Municipal & 75 & 2,883 & 0,294 & 2,303 & 3,463 \\
Subvencionado & 211 & 2,903 & 0,174 & 2,560 & 3,246 \\
Particular & 31 & 1,316 & 0,486 & 0,359 & 2,273 \\
Sexo por Tipo de Colegio & & & & \\
F-Municipal & 32 & 2,645 & 0,444 & 1,771 & 3,519 \\
F-Subvencionado & 93 & 2,777 & 0,260 & 2,264 & 3,290 \\
F-Particular & 10 & 1,269 & 0,796 & $-0,297$ & 2,836 \\
M-Municipal & 43 & 3,122 & 0,386 & 2,361 & 3,883 \\
M-Subvencionado & 118 & 3,029 & 0,231 & 2,574 & 3,484 \\
M-Particular & 21 & 1,363 & 0,551 & 0,2775 & 2,449 \\
\hline
\end{tabular}

Tabla IV. Análisis de varianza para ceod en la población escolar de niños que cursan segundo básico en Temuco.

\begin{tabular}{lccccc}
\hline Fuente & $\begin{array}{c}\text { Suma } \\
\text { Cuadrados }\end{array}$ & GI. & $\begin{array}{c}\text { Cuadrado } \\
\text { Medio }\end{array}$ & Razón F & Valor P \\
\hline $\begin{array}{l}\text { Covariables } \\
\text { IHOS }\end{array}$ & 9,117 & 1 & 9,117 & 1,45 & 0,230 \\
Efectos principales & 3,052 & 1 & 3,052 & 0,48 & 0,487 \\
A: Sexo & 60,875 & 2 & 30,43 & 4,83 & $0,008^{* *}$ \\
B: Tipo de Colegio & & & & & \\
Interacciones & 0,972 & 2 & 0,486 & 0,08 & 0,925 \\
AB & 1955,2 & 310 & 6,307 & & \\
Residuos & 2051,3 & 316 & & & \\
Total Corregido & & & & & \\
\hline
\end{tabular}

El análisis de regresión múltiple para explicar el comportamiento del índice ceod en función de la Fluorosis (Índice de Dean) e Higiene Oral mediado por los factores sexo y tipo de colegio no mostró relación entre las variables. 
OLIVARES-KELLER, D.; ARELLANO-VALERIA, M. J.; CORTÉS, J. \& CANTíN, M. Prevalencia y severidad de fluorosis dental y su asociación con historia de caries en escolares que consumen agua potable fluorurada en Temuco, Chile. Int. J. Odontostomat., 7(3):447-454, 2013.

Tabla V. Resumen de Medias por Mínimos Cuadrados y EE (Error estándar) e intervalos de confianza al 95\%, para el índice COPD.

\begin{tabular}{lccccc}
\hline Variable & Casos & Media & EE & Límite Inf. & Límite Sup. \\
\hline Sexo & & & & & \\
Femenino (F) & 135 & 0,175 & 0,059 & 0,059 & 0,292 \\
Masculino (M) & 182 & 0,140 & 0,044 & 0,052 & 0,227 \\
$\begin{array}{l}\text { Tipo de Colegio } \\
\text { Municipal }\end{array}$ & & & & \\
Subvencionado & 211 & 0,133 & 0,032 & 0,069 & 0,197 \\
Particular & 31 & 0,139 & 0,091 & $-0,040$ & 0,318 \\
Sexo por Tipo de Colegio & & & & & \\
F-Municipal & 32 & 0,278 & 0,083 & 0,114 & 0,442 \\
F-Subvencionado & 93 & 0,132 & 0,048 & 0,036 & 0,228 \\
F-Particular & 10 & 0,116 & 0,149 & $-0,177$ & 0,410 \\
M-Municipal & 43 & 0,124 & 0,072 & $-0,018$ & 0,266 \\
M-Subvencionado & 118 & 0,134 & 0,043 & 0,0491 & 0,219 \\
M-Particular & 21 & 0,161 & 0,103 & $-0,042$ & 0,365 \\
\hline
\end{tabular}

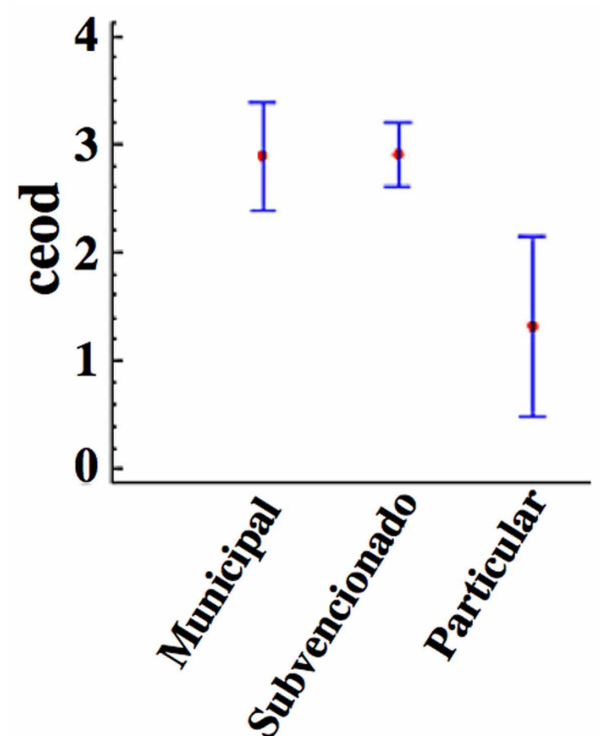

Fig. 1. Medias de Bonferroni para los índices ceod según tipo de establecimiento educacional Municipal, Subvencionado y Particular con un IC de $95 \%$.

\section{DISCUSIÓN}

Evaluamos el efecto de la fluoruración del agua potable en los niveles de prevalencia y severidad de fluorosis dental en escolares residen- tes de la ciudad de Temuco, a 8 años desde que se implementó su aplicación, quiénes estuvieron expuestos a esta medida masiva y equitativa de prevención de caries durante todo su desarrollo dentario. La prevalencia de fluorosis fue elevada, afectando al $53 \%$ de la población, y en estos, más del $50 \%$ presentó un grado de severidad cuestionable, muy leve o leve, con implicancias principalmente estéticas.

En los últimos años se ha observado un incremento significativo de esta patología en zonas donde se ha fluorurado el agua potable con fines preventivos con concentraciones permitidas y controladas (Beltrán-Valladares et al., 2005; Urbina et al., 1997; Azpeitia-Valadez et al., 2008; Levy; Soto et al.). En el contexto mundial, su prevalencia se sitúa entre el $7,7 \%$ y $80,7 \%$ en áreas donde se cuenta con agua fluorurada, mientras que en áreas con disponibilidad de flúor por otras fuentes, entre ellas la sal, se sitúa entre $2,9 \%$ y $42 \%$, donde la severidad de tipo leve es más frecuente (Beltrán-Valladares et al.).

Para la OMS, la tasa estándar de fluoruros en el agua potable oscila entre 0,5 y 1 ppm (Seppä, 2001), considerando 1 ppm como la concentración óptima o recomendada, con el máximo efecto de protección frente a la caries y el mínimo riesgo de fluorosis dental (Bailey et al., 2006; Kaseva, 2006; Tamer et al., 2007). En Chile, la concentración óptima oscila entre 0,6 y 1,0 mg/L (Allende et al., 2008). El riesgo de desarrollar fluorosis dental aumenta cuando el agua excede la concentración máxima permitida (que varía según región o zona geográfica), sobre todo en presencia de concentraciones entre 2 a 3 ppm durante los períodos críticos de la formación dentaria (Allende et al.; Narwaria et al., 2013). En la ciudad de Temuco el agua potable se fluorura desde el año 2004, con una concentración de 0,9 ppm (Superintendencia de Servicios Sanitarios, 2010). A pesar de ello nuestros resultados mostraron una elevada prevalencia de fluorosis dental. Según Mella et al. (1992) el consumo promedio de flúor en meno- 
res de 12 años expuestos a concentraciones de $1 \mathrm{ppm}$ equivalen a $0,05 \mathrm{mg}$ de flúor, la mitad de los miligramos necesarios para generar alguna alteración del esmalte. Por tanto la concentración de flúor en el agua potable de Temuco no explicaría por sí sola la elevada prevalencia de fluorosis dental, siendo necesario considerar otras fuentes de exposición y administración, muchas de las cuales utilizan el agua (ya fluorada) como vehículos para ingresar al organismo.

La administración sistémica de fluoruros, también considera el consumo de alimentos fluorurados naturalmente (pescado, té, espinacas, tomate, entre otros) y de manera artificial como la sal y leche (Gómez et al., 2002); esta última administrada en establecimientos educacionales rurales de Chile para dar cobertura a zonas que no poseen agua potable fluorurada. Además, otra posible fuente de administración es la ingesta accidental de dentífricos $u$ otros productos de higiene oral que incorporen fluoruros (Mattos-Vela et al., 2013). Estas fuentes de fluoruros serían potenciadas con el agua ya intervenida. Por tanto, la fluorosis dental estaría influenciada por factores como la i) concentración de flúor, ii) cantidad ingerida, iii) frecuencia de ingesta y iv) etapa de desarrollo dentario.

En Chile, pocos estudios evidencian el impacto de la fluoruración del agua sobre la salud oral de la población. Ballesteros \& Olave (2004) reportaron una prevalencia de fluorosis dental del $3,1 \%$ en una población de 159 escolares de segundo básico en la ciudad de Temuco, previo a la intervención del agua con fluoruros, lo que es comparativamente inferior al $53 \%$ reportado en nuestro estudio realizado a 8 años de implementada la medida. Esta diferencia concuerda con lo reportado por Yévenes et al. (2011) quienes analizaron los efectos de la fluoruración del agua en la Región Metropolitana y describieron un aumento de la prevalencia de fluorosis dental desde $4,2 \%$ al $32,2 \%$ después de 8 años de implementada la medida. Ya en el año 1992, un estudio piloto realizado por Mella et al. sobre 118 escolares con al menos 6 años de residencia en lugares con diferentes concentraciones de flúor, mostró un $54 \%$ de prevalencia de fluorosis dental en Chile, alertando la necesidad de vigilancia epidemiológica para la administración de fluoruros. Urbina et al. estudiaron la fluorosis dental en seis regiones de Chile mediante el Índice de Dean (tradicional), con una prevalencia de $3,4 \%$ para edades entre 6 y 8 años, y grado de severidad cuestionable en $0,9 \%$ de los casos, muy leve en $9,8 \%$, leve en $3,9 \%$ y moderada $1,2 \%$, con ausencia de casos severos.

En otros países de Latinoamérica como México, la prevalencia de fluorosis alcanza el $56,3 \%$ en niños entre 6 y 9 años de la ciudad Campeche, con una severidad muy leve, leve y severa en el $45 \%, 10 \%$ y $1,3 \%$ de los casos, respectivamente (Beltrán-Valladares et al.), mientras que los escolares de 12 años del Municipio de Nezahualcóyotil alcanzan el $70,84 \%$, con una severidad dudosa y muy leve principalmente (Aguilar et al., 2007). En Venezuela, alcanza el 16,6\% entre escolares de 8 y 12 años, con severidad muy leve $(8,5 \%)$ principalmente (Montero et al., 2007). En zonas de fluorosis endémica como India, China y África (Budipramana et al., 2002; Firempong et al., 2007; Tellez et al., 2011; Casanova et al., 2013), donde las fuentes de agua presentan de manera natural altas concentraciones de fluoruros, la prevalencia va desde el $30 \%$ hasta $100 \%$.

Desde la implantación de esta medida preventiva, la situación de caries en niños de 7 años en Temuco ha disminuido desde índices ceod de 5,89 y COPD de 1,51 (Ballesteros \& Olave) hasta índices ceod de 2,37 y COPD de 0,16 (estudio actual), especialmente en dentición primaria, lo que reafirma la eventual efectividad de esta medida (Yévenes et al.; Brasil. Ministério da Saúde, 2004; Menghini et al., 2003). Sin embargo, la reducción de caries no puede solamente ser atribuida a la incorporación de fluoruros en el agua, ya que elementos como la educación, alimentación no cariogénica, hábitos de higiene, acceso a la atención odontológica, así como la incorporación de fluoruros por otros medios, están involucrados.

Es relevante tener en cuenta la disminución de estos índices, y necesario continuar con su estudio para evaluar la mantención de fluoruración del agua potable como medida de salud pública, ya que la Secretaría Regional Ministerial de Salud respectiva determinará por resolución los servicios que a su juicio deban fluorurar el agua; esta resolución debe ser emitida cuando la población a ser beneficiada, presente altos indicadores de caries dental (prevalencia y severidad), y el nivel de fluoruros naturales presentes en el agua, sea insuficiente para prevenir caries (menor a 0,5 mg/L) (Allende et al.).

Nuestros resultados podrían presentar limitaciones por sesgos vinculados a la posible inclusión de escolares que viven en zonas rurales sin agua potable fluorurada y asisten a establecimientos urbanos, o que migraron a zonas con agua potable fluorurada entre el año 2004 y 2012. Esta información no pudo ser controlada. Tampoco fue posible estimar si hubo sobre exposición a fluoruros durante la odontogénesis o mediante campañas de aplicación de flúor escolar, así como corroborar la concentración específica de fluoruros en el agua desde el año 2004 hasta el momento del estudio. 
OLIVARES-KELLER, D.; ARELLANO-VALERIA, M. J.; CORTÉS, J. \& CANTíN, M. Prevalencia y severidad de fluorosis dental y su asociación con historia de caries en escolares que consumen agua potable fluorurada en Temuco, Chile. Int. J. Odontostomat., 7(3):447-454, 2013.

Finalmente, se deben considerar las consecuencias estéticas causadas por la fluorosis en los dientes, especialmente en el sector anterior de la boca, ya que los niños son capaces de detectar este defecto como algo anormal y en ocasiones vergonzoso en sus dientes (sobre todo al sonreír e interactuar con otros niños) (Tellez et al.), afectándolos independientemente de su nivel socioeconómico o cultural.

\section{CONCLUSIÓN}

En Temuco al 2012, la prevalencia de fluorosis dental afecta al $53 \%$ de los niños de siete años, con una severidad de tipo cuestionable, muy leve y leve en más del $50 \%$ de los casos.

Después de 8 años implementada la fluoruración del agua potable como política de salud pública, observamos una disminución del daño acumulado por caries.
Sin embargo, la fluorosis dental aumentó, sin asociarse de manera significativa con la historia de caries.

Es necesario realizar nuevos estudios de vigilancia epidemiológica para conocer la situación y realidad particular en otras ciudades de chile, con el fin de implementar planes de monitoreo del agua potable, reevaluando la concentración de fluoruros necesaria, ya que esta es dependiente para cada región. Así, se podría evaluar el efecto de esta medida masiva de prevención en salud que trae consigo una inversión estatal considerable, además de un riesgo potencial, a fin de prevenir el incremento en la prevalencia y formas severas de fluorosis.

\section{AGRADECIMIENTOS}

Los autores agradecen la colaboración de los Directores, Personal y Apoderados de los establecimientos Educacionales participantes.

OLIVARES-KELLER, D.; ARELLANO-VALERIA, M. J.; CORTÉS, J. \& CANTíN, M. Prevalence and severity of dental fluorosis and its association with history of dental caries in schoolchildren consuming fluoridated drinking water in Temuco, Chile. Int. J. Odontostomat., 7(3):447-454, 2013.

ABSTRACT: Fluoridation of drinking water, began to be implemented in 1950's in Chile to prevent the main problem of oral health in the population, dental caries. It has been consistently applied in the country and Temuco city since 2004. However, intake of fluorides in critical periods of tooth development excessively produces dental fluorosis. The aim was to determine the prevalence and severity of dental fluorosis in elementary schoolchildren consuming fluoridated drinking water in the city of Temuco, Chile and its association with the history of dental caries. A descriptive and explanatory epidemiological study with a stratified random sample of 7-years schoolchildren of private, subsidized and municipal elementary schools in Temuco city was performed. On a sample of 317 children, Dean's index and history of caries was determined. The prevalence of dental fluorosis was $53.31 \%$ ( 169 children), and severity rate was questionable, very mild and mild in $31.36 \%, 42.6 \%$ and $22.4 \%$ of cases, respectively. In $3.5 \%$ was moderate. No significant association between fluorosis and history of dental caries was observed. In Temuco, the prevalence of dental fluorosis in 7 -years children is high, with severity of questionable, very mild and mild type in $50 \%$ of cases. After 8 years of implementing the fluoridation of drinking water, there was a decrease of dental caries damage but increased dental fluorosis in the population.

KEY WORDS: dental fluorosis, grades of fluorosis, dental caries, drinking water, Dean's Index.

\section{REFERENCIAS BIBLIOGRÁFICAS}

Aguilar, R. Y. P. \& De la Cruz, C. D. Estudio Epidemiológico de Fluorosis Dental en el Municipio de Nezahualcóyotl, Estado de México. Vertientes, 10(1-2):33-7, 2007.

Allende, M.; Fernández, O.; Gómez, S.; Horta, M.; Humeres, P.; Pastore, C.; Vicentella, L.; Villa, A.; Yevenes, I.; Zillmann, G.; Weitz, A. \& Sandoval, S. Normas de uso de Fluoruros en la prevención odontológica. Norma General Técnica № 105, Santiago, Ministerio de Salud, 2008.

Aoba, T. \& Fejerskov, O. Dental fluorosis: chemistry and biology. Crit. Rev. Oral Biol. Med., 13(2):155-70, 2002.
Azpeitia-Valadez, M. de L.; Rodríguez-Frausto, M. \& SánchezHernández, M. A. Prevalencia de fluorosis dental en escolares de 6 a 15 años de edad. Rev. Med. Inst. Mex. Seguro Soc., 46(1):67-72, 2008.

Bailey, K.; Chilton, J.; Dahi, E.; Lennon, M.; Jackson, P. \& Fawell, J. World Health Organisation. Fluorides in drinkingwater. WHO drinking-water quality series. London, IWA Publishing, 2006.

Ballesteros, A. \& Olave, P. Estudio de prevalencia de caries y fluorosis en escolares de segundo básico, en relación a la fluoración del agua potable en la ciudad de Temuco, IX Región. Proyecto de Investigación para optar al grado de Licenciado en Odontología con Mención en Prevención. Temuco, Universidad de La Frontera, 2004. 
Beltrán-Valladares, P. R.; Cocom-Tun, H.; Casanova-Rosado, J. F.; Vallejos-Sánchez, A. A.; Medina-Solís, C. E. \& Maupomé, G. Prevalencia de fluorosis dental y fuentes adicionales de exposición a fluoruro como factores de riesgo a fluorosis dental en escolares de Campeche, México. Rev. Invest. Clín., 57(4):532-9, 2005.

Brasil. Ministério da Saúde. Projeto SB: Condições de Saúde Bucal da população Brasileira 2002-2003. Brasília, Ministério da Saúde, 2004.

Budipramana, E. S.; Hapsoro, A.; Irmawati, E. S. \& Kuntari, S. Dental fluorosis and caries prevalence in the fluorosis endemic area of Asembagus, Indonesia. Int. J. Paediatr. Dent., 12(6):415-22, 2002.

Buzalaf, M. A. \& Levy, S. M. Fluoride intake of children: considerations for dental caries and dental fluorosis. Monogr. Oral Sci., 22:1-19, 2011.

Casanova, R. A. J.; Medina, S. C. E.; Casanova, R. J. F.; Vallejos, S. A. A.; de la Rosa, S. R.; Mendoza, R. M.; Villalobos, R. J. J. \& Maupomé, C. G. Prevalencia de fluorosis dental en ocho cohortes de mexicanos nacidos durante la instauración del Programa Nacional de Fluoruración de la Sal Doméstica. Gac. Méd. Méx., 149(1):27-35, 2013.

de Carvalho, R. B.; Medeiros, U. V.; dos Santos, K. T. \& Pacheco Filho, A. C. Influence of different concentrations of fluoride in the water on epidemiologic indicators of oral health/disease. Cien. Saude Colet., 16(8):3509-18, 2011.

Firempong, C.; Nsiah, K.; Awunyo-Vitor, D. \& Dongsogo, J. Soluble fluoride levels in drinking water-a major risk factor of dental fluorosis among children in Bongo community of Ghana. Ghana Med. J., 47(1):16-23, 2013.

Gómez, S. G.; Gómez, S. D. \& Martín, D. M. Flúor y Fluorosis dental. Pautas para el consumo de dentífricos y aguas de bebida en Canarias. Santa Cruz de Tenerife, Dirección General de Salud Pública. Servicio Canario de la Salud, 2002.

Kaseva, M. E. Contribution of trona (magadi) into excessive fluorosis--a case study in Maji ya Chai ward, northern Tanzania. Sci. Total Environ., 366(1):92-100, 2006.

Levy, S. M. An update on fluorides and fluorosis. J. Can. Dent. Assoc., 69(5):286-91, 2003.

Mattos-Vela, M. A.; Carrasco-Loyola, M. B. \& Valdivia-Pacheco, S. G. Nivel de Conocimiento sobre Pasta Dental Fluorada en Padres y Profesores de Preescolares. Int. J. Odontostomat., 7(1):17-24, 2013.

Mella, O. S.; Atalah, S. E.; Aranda, C. W. \& Montagna, M. R. Fluorosis dental endémica en Chile: estudio piloto. Rev. Med. Chil., 120(8):866-71, 1992.
Menghini, G.; Steiner, M.; Marthaler, T.; Helfenstein, U.; Brodowski, D.; Imfeld, C.; Weber, R. \& Imfeld, T. Caries prevalence among students in 16 Zurich districts in the years 1992 to 2000. Schweiz. Monatsschr. Zahnmed., 113(3):267-77, 2003.

Montero, M.; Rojas-Sanchez, F.; Socorro, M.; Torres, J. \& Acevedo, A. M. Experiencia de caries y fluorosis dental en escolares que consumen agua con diferentes concentraciones de fluoruro en Maiquetía, Estado Vargas, Venezuela. Invest. Clín., 48(1):5-19, 2007.

Narwaria, Y. S. \& Saksena, D. N. Prevalence of dental fluorosis among primary school children in rural areas of Karera Block, Madhya Pradesh. Indian J. Pediatr., 80(9):718-20, 2013.

Seppä, L. The future of preventive programs in countries with different systems for dental care. Caries Res., 35(Suppl. 1):26-9, 2001.

Soto, L.; Tapia, R.; Jara, G. \& Rodríguez, G. Diagnóstico Nacional de Salud Bucal del Niño de 6 años. Santiago, Gobierno de Chile, Ministerio de Salud, 2007.

Superintendencia de Servicios Sanitarios. Estudio Tarifario Empresa Aguas Araucanía S.A., Periodo 2011 - 2016. Informe Final. Santiago, Gobierno de Chile, 2010.

Tamer, M. N.; Kale Köroglu, B.; Arslan, C.; Akdogan, M.; Köroglu, M.; Cam, H. \& Yildiz, M. Osteosclerosis due to endemic fluorosis. Sci. Total Environ., 373(1):43-8, 2007.

Tellez, M.; Santamaria, R. M.; Gomez, J. \& Martignon, S. Dental fluorosis, dental caries, and quality of life factors among schoolchildren in a Colombian fluorotic area. Community Dent. Health, 29(1):95-9, 2012.

Urbina, T.; Caro, J. \& Vicent, M. Caries y fluorosis en niños de 6 a 8 años y 12 años. Santiago de Chile, Ministerio de Salud, Departamento de Estadísticas e Información, 1997.

Yévenes, I.; Zillmann, G.; Muñoz, A.; Aranda, W.; Echeverría, S.; Hassi, J.; Maass, P. \& Salazar, M. Caries and fluorosis in the Santiago metropolitan region in Chile:The impact of the fluoridation of the water. Rev. Odonto Cienc., 26(2):109-15, 2011.

Dirección para Corresponcia:

María José Arellano Valeria

Escuela de Odontología

Facultad de Odontología

Universidad de La Frontera

Recibido : 19-07-2013

Casilla 54-D

Aceptado: 22-10-2013

Temuco-CHILE

Email: mariajosearellano22@outlook.com 